Case Report

\title{
Rosai-Dorfman disease presenting in a child as mediastinal lymphadenopathy
}

\author{
Jeyarani G. ${ }^{1}$, Sneha LM², Jayaraman $D^{3}$., Gunabooshanam B. ${ }^{4}$, Periakaruppan G. ${ }^{5}$, Kumaravel C. ${ }^{6}$ \\ ${ }^{1}$ Dr. Gracelin Jeyarani, MD Pediatrics, Final Year, Postgraduate, ${ }^{2}$ Dr. Latha M. Sneha, Associate Professor, Division of \\ Pediatric Hemato Oncology, ${ }^{3}$ Dr. Dhaarani Jayaraman, Assistant Professor, Division of Pediatric Hemato Oncology, \\ ${ }^{1,2,3}$ Authors are affiliated with the Department of Pediatrics, ${ }^{4}$ Dr. Barathi Gunabooshanam, Assistant Professor, Department of \\ Pathology, ${ }^{5}$ Dr. Gokulakrishnan Periakaruppan, Associate Professor, Department of Radiology, ${ }^{6}$ Dr. Chitra Kumaravel, Intern, \\ Department of Pediatrics, Sri Ramachandra Institute of Higher Education and Research, Chennai, Tamil Nadu, India
}

Corresponding Author: Dr. Latha M. Sneha, Associate Professor, Division of Pediatric Hemato Oncology, Department of Pediatrics, Sri Ramachandra Institute of Higher Education and Research, Chennai, Tamil Nadu, India. E-mail: shreeben@gmail.com

\begin{abstract}
Rosai- Dorfman disease (RDD) or Sinus Histiocytosis with massive lymphadenopathy (SHML) is a rare benign disease with infiltration of lymph nodes or extranodal sites by non malignant histiocytes. It generally presents with fever, painless cervical lymphadenopathy and eleveated inflammaotory markers. Sometimes, it may be associated with night sweats and weight loss mimicking lymphoma. Forty per cent, present with extra nodal manifestations and $10 \%$ have systemic involvement. Though the disease has a predilection for lymph nodes in head and neck region, it can affect any organ. The common extra nodal sites are skin and soft tissue, central nervous system, upper respiratory tract and gastrointestinal tract. Immune dysfunction and viral infections like HPV 6, EBV, parvo virus B19 are reported to be associated with the disease. RDD typically occurs in childhood and early adulthood. Histologially, characteristic finding of emperipolesis is seen. Diagnois is confirmed by Immunohistochemistry with S100 and CD68 positivity. Most of them do not require treatment as it is usually self-limiting. Steroids, surgery, chemotherapy and radiotherapy are considered in refractory disease with systemic involvement or vital organ involvement. We report a case of 4 year old boy who presented with massive bilateral cervical and mediastinal lymphadenopapthy and had complaints of breathing difficulty while lying down since last 3 months. Biopsy was confirmative of Rosai-Dorfman disease and he had a near complete response after 3 months of steroids and is symptom free for the past 1 year.
\end{abstract}

Keywords: Mediastinal lymphadenopathy, Cervical lymphadenopathy, Child, Rosai-Dorfman disease

\section{Introduction}

With a childhood predilection, Sinus Histocytosis with Massive Lymphadenopathy (SHML) or Rosai-Dorfman disease (RDD), is a rare and benign cause of lymphadenopathy in the first decade of life. It is a nonlangerhans-cell histiocytic proliferative disease of unkown etiology [1]. Patients usually present with bilateral painless cervical lymphadenopathy that usually undergoes spontaneous remission.

They may also present with systemic symptoms like fever, night sweats, malaise and weight loss which are attributed to the enhanced production of monokines by histiocytes [2]. Cervical lymphadenopathy is commonly involved in

Manuscript received: $18^{\text {th }}$ November 2019

Reviewed: $28^{\text {th }}$ November 2019

Author Corrected: $4^{\text {th }}$ December 2019

Accepted for Publication: $7^{\text {th }}$ December 2019
$90 \%$ of cases and axillary, para aortic, inguinal and mediastinal lymph nodes are less frequently affected. About $40 \%$ of the cases present with extranodal manifestations and $10 \%$ are associated with soft tissue involvement which can be an isolated finding without any associated lymphadenopthy [3].

Though any system can be affected, the most common extranodal sites are skin and soft tissue, upper respiratory tract, orbit and eye, bone and salivary gland. Simultaneous involvement of the multiple extranodal sites are also reported [2].

The pathogenesis of RDD has not been established and is assumed to be an aberrant response to an unspecified antigen possibly an infective agent. 
Case Report

\section{Case Report}

A 4 year old developmentally normal boy child, first born to non consaguineous parents was brought with the complaints of progressively increasing multiple bilateral neck swellings, fever and cough since last three months. The swelling was slowly progressive in nature and was not associated with pain or any skin changes or difficulty in swallowing.

The parents gave a history of intermittent fever for the last three months that was not associated with skin rash or night sweats or weight loss. The cough was not associated with any increased work of breathing. The boy also has been complaning of difficulty in breathing while lying down in supine position since last 3 weeks. There was no history of contact with tuberculosis in the family.

On examination, his height and weight were within $50^{\text {th }}$ to $75^{\text {th }}$ centile. Respiratory rate was $20 / \mathrm{min}$ and Heart rate was $96 / \mathrm{min}$. No organomegaly was palpable. Multiple massive bilateral cervical lymph nodes were palpable - largest was $8 \mathrm{cms} x 9 \mathrm{cms}$ in the right side and 10x $11 \mathrm{cms}$ in the left side (Figure 1). Bilateral inguinal nodes were also palpable, largest being $2 \mathrm{cmsx} 3 \mathrm{cms}$ in the right side.

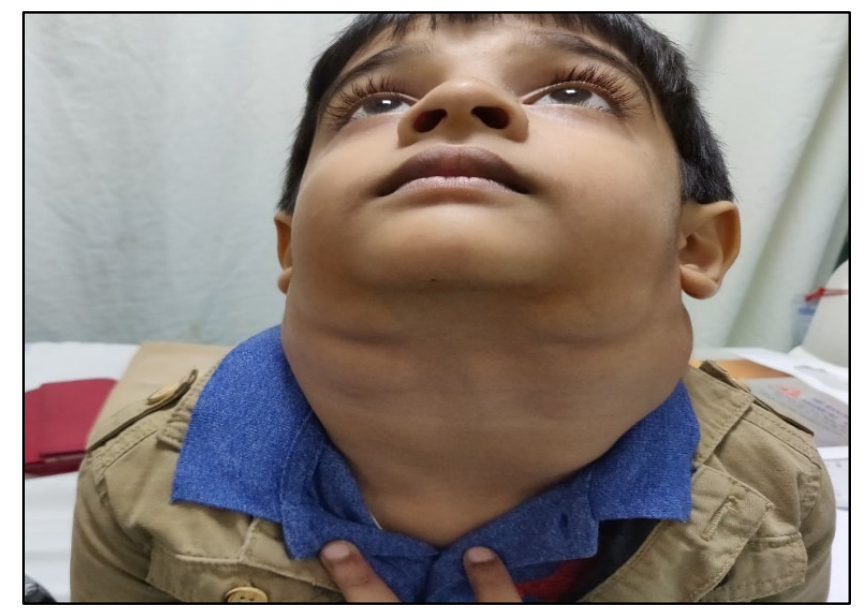

Fig-1: Bilateral massive cervical lymphadenopathy in level I, II, III, IV and V levels.

His hemoglobin was $8.7 \mathrm{~g} / \mathrm{dl}$ and total leukocyte counts was 14,000 cells/mm3. The ESR was $70 \mathrm{~mm}$ in first hour. Liver and renal function tests, serum electrolytes were normal. The child underwent whole body PET CT and found to have cervical, mediastinal and inguinal lymphadenopathy (Figure 2).

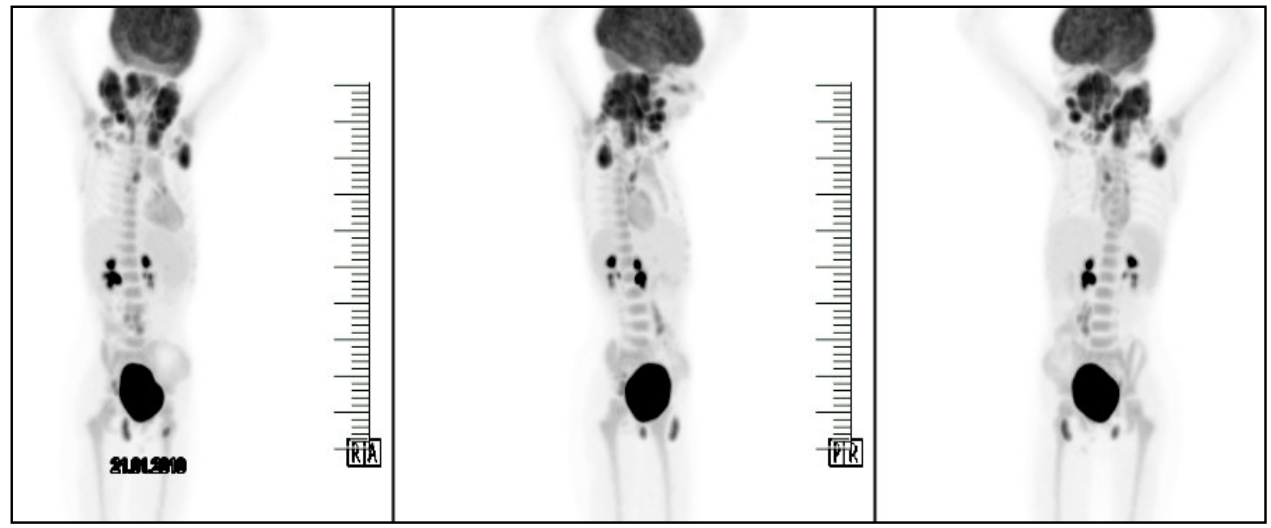

Fig-2: PET/CT scan showing multiple FDG avid nodes in: bilateral cervical, tonsillar, axillary, pretracheal, subcarinal, prevascular, right hilar and bilateral inguinal nodes. Largest of these nodes seen in left cervical region measuring $9.5 \mathrm{x}$ $5.9 \mathrm{~cm}$ with SUV max of 7.82 (conglomerate nodal mass of level 1b, II, III, IV and V).

The child underwent exicison biopsy of the largest left side cervical lymph node which revealed effaced architecture and emperipolesis (Figure 3). 
Case Report

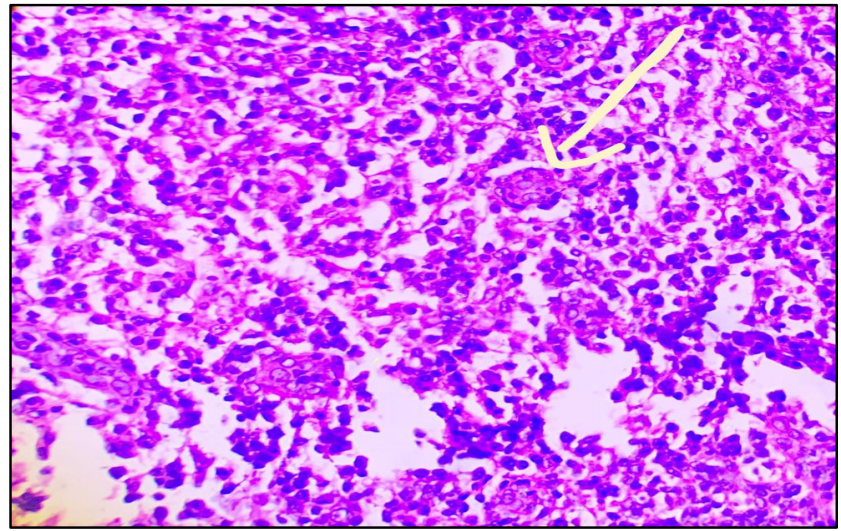

Fig-3: Lymphnode with effaced architecture showing dilated sinus filled with histiocytes. The histiocytes show intact lymphocytes, plasma cells and neutrophils are the cytoplasm - resembling emperipolesis.

After the diagnosis of Rosai-Dorfman was confirmed by S-100 positivity by IHC in the biopsy block, child was initially given Inj methyl prednisolone in view of massive mediastinal lymphadenopathy causing breathing difficulty and later changed to oral prednisolone $(1 \mathrm{mg} / \mathrm{kg} /$ day). There was a significant reduction in the size of the cervical lymph nodes in 4 weeks and the dose was tapered to $25 \%$ reduction every 2 weeks.

PET CT repeated after 6 months showed a near complete response (Figure 4) and he was started on maintenance therapy with 6 Mercaptopurine and Methotrexate for 1 year.

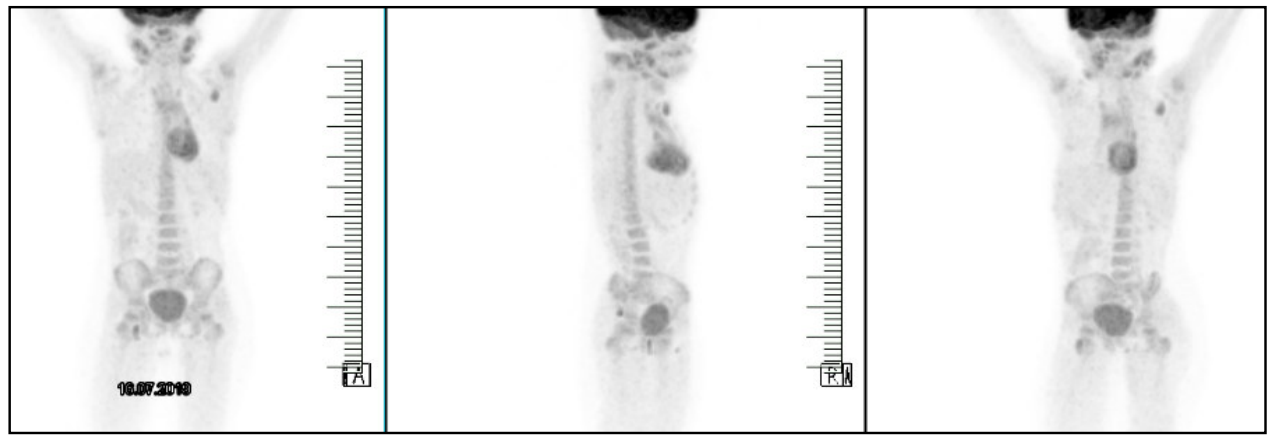

Fig-4: follow up PET/CT scan after six months show significant resolution of bilateral cervical, axillary and inguinal FDG avid nodes. Largest residual node measures $2.0 \times 1.7 \mathrm{~cm}$ with SUV max of 2.99 in left cervical region. Complete metabolic response seen in bilateral tonsillar, pretracheal, subcarinal, prevascular and right hilar regions.

\section{Discussion}

Rosai-Dorfman disease (RDD), also known as sinus histiocytosis with massive lymphadenopathy (SHML), is a rare, benign, idiopathic, non-Langerhans cell histiocytic proliferative disorder. The clincial course is chronic with periods of remission and exacerbation of lymphadenopathy and there are no standard guidelines for duration of treatment. Twenty-five per cent of cases present with systemic involvement [4]. CNS involvment is seen in less than $5 \%$ and the presentation is limited to brain or spinal cord and is not associated with lymphadenopathy [5]. Mediastinal lymphadenopathy is a feature of RDD, but only few pediatric cases are reported. In cases of lymphadenopathy, the other etiologies to be ruled out are, $M$. tuberculosis and non-tuberculous mycobacteria, sarcoidosis, fungal infections, lymphoma, HIV associated non-hodgkins and Kaposi sarcoma.
Due to the fact that RDD may affect every organ and system, symptoms vary depending on the localisation of the disease. The most frequent clinical presentation of RDD is massive, bilateral, painless cervical lymphadenopathy accompanied by fever [4]. In most cases the patients are in good general condition, although night sweats and weight loss may also be observed. As a result, RDD can sometimes mimic lymphoma.

The etiology of RDD is still unclear. Multiple stimuli like post infectious conditions, auto immune diseases, immune deficiency and hematological malignancies might result in cytokine mediated migration of monocytes resulting in histioytes accumulation. Studies have reported an association with HHV 6, parvovirus B19, CMV, EBV, simian virus 40, Brucella and Klesiella [4]. 
Case Report

Laboratory findings in RDD are non-specific. The most frequent features include leukocytosis, elevated ESR and CRP levels, and polyclonal gammopathy. Normochromic normocytic anemia and elevated serum ferritin levels have also been described. Presence of immunological abnormalities often leads to ufavourable disease outcome.

They may present with rheumatoid factor, hypergammaglobulinemia, antinuclear antibodies, reversal of CD4/CD8 ratio, low serum IgA levels, decreased mitogenic responses and cutaneous anergy [6].

RDD is histologically seen as non-malignant polymorphous cell proliferation of histiocytic or phagocytic cells within the lymph node sinuses and lymphatics in extranodular sites (rosai-Dorfman cells) in the background of mixed inflammatory infiltrate, consisting of moderately abundant plasma cells and lymphocytes $[7,8]$. The characteristic diagnostic feature of RDD is lymphocytophagocytosis or emperipolesis presence of intact lymphocytes within the histiocytic cytoplasm. Extra nodal RDD exhibits more fibrosis and lesser histiocytes with emperipolesis.

Due to its propensity for spontaneous remission, "wait and watch" is normally followed. In cases of complications like upper airway obstruction and vital organ involvement, treatment becomes necessary. Waxing and waning of lymphadenopathy is very common and duration of treatment is not standardized. The most common therapeutic drugs are steroids and chemotherapy with alkylating agents, anthracyclines and antimetabolites in steroid refracory cases [9]. Foucar et al reported stable disease inn 54\% and spontaneous regression in $21 \%$ and progressive disease in $1 \%$ of his study population [4]. The lymph nodes have been reported to persist for as long as three years and steroid dependene has also been documented [10].

\section{Conclusion}

Rosai-Dorfman disease should be ruled out in cases of massive bialteral cervical lymphadenopathy.

Funding: No funding sources

Conflict of interest: None declared

Ethical Approval: Not required

\section{References}

1. Gaitonde S. Multifocal, extranodal sinus histio- cytosis with massive lymphadenopathy: an overview. Arch Pathol Lab Med. 2007;131(7):1117-1121.

2. Zhao M, Li C, Zheng J, Yu J, Sha H, Yan M, et al. Extranodal Rosai Dorfman disease involving appendix and mesenteric nodes with a protracted course: report of a rare case lacking relationship to IgG4-related disease and review of the literature. Int J Clin Exp Pathol. 2013; 6(11): 2569-2577.

3. McClain KL, Natkunam Y, Swerdlow SH. Atypical cellular disorders. Hematology Am Soc Hematol Educ Program 2004;1:283-296. doi: https://doi.org/10. 1182/ asheducation-2004.1.283.

4. Foulard E, Rosa J, Dorman RF. Sinus histocytosis with massive lymphadenopathy (Rosia- Dorfman disease): Review of the entity: Semin Diagn Patho. 1990;7(1):19-73

5. Wu M, Anderson AE, Kahn LB. A report of intracranial Rosai-Dorfman disease with literature review. Ann Diagn Pathol. 2001; 5(2): 96-102. doi: https://doi.org/10.1053 /adpa. 2001.23027.

6. Maennle DL, Grierson HL, Gnarra DG. Weisenburger DD. Sinus histiocytosis with massive lymphadenopathy: a spectrum of disease associated with immune dysfunction. Pediatr Pathol.1991;11(3):399-412.

7. Rodriguez-Galindo C, Helton KJ, Sánchez ND, Rieman M, Jeng M, Wang W. Extranodal Rosai-Dorfman disease in children. J Pediatr Hematol Oncol. 2004;26(1):19-24.

8. Juskevicius R, Finley JL. Rosai-Dorfman disease of the parotid gland: cytologic and histopathologic findings with immunohisto- chemical correlation. Arch Pathol Lab Med. 2001; 125(10):1348-1350.

9. Horneff G, Jurgens H, Hort W, Karitzky D, Gobel U. Sinus histiocytosis with massive lymphadenopathy (RosaiDorfmann disease): response to methotrexate and mercaptopurine. Med Pediatr Oncol.1996;27(3):187-192.

10. Antonius JI, Farid SM, Baez-Giangreco A. Steroid responsive Rosai-Dorfman disease. Pediatric Hematol Oncol 1996;13(6):563-570. doi: https://doi.org/10. 3109/ 08880019609030873 .

\section{How to cite this article?}

Jeyarani G, Sneha LM, Jayaraman D., Gunabooshanam B, Periakaruppan G, Kumaravel C. Rosai-Dorfman disease presenting in a child as mediastinal lymphadenopathy. Int J Pediatr Res.2019;6(12):625-628.doi:10.17511/ijpr.2019.i12.07 